\title{
Emergency department procedural sedation practice in Cape Town, South Africa
}

\author{
P. W. Hodkinson • M. F. M. James • L. A. Wallis
}

Received: 6 October 2008 / Accepted: 23 February 2009/Published online: 4 June 2009

(C) Springer-Verlag London Ltd 2009

\begin{abstract}
Background There are no general policies or protocols for procedural sedation in the emergency department and no literature on present practice in South Africa.

Aims To investigate procedural sedation (PS) practice in adults in emergency departments (EDs) in Cape Town, South Africa.

Methods A cross-sectional descriptive study was performed by interviewing all ED managers and ED doctors in Cape Town meeting the criteria (open $24 \mathrm{~h}$ a day, staffed by fulltime doctors, seeing adult patients and doctors who practice primarily emergency medicine and have performed at least one PS in the last 3 months).

Results Data were collected from 13 units (5 public, 8 private) and 76 clinicians (48 public, 28 private). PS facilities are generally good in the private sector, but poor in the public sector (lacking in equipment, staff and protocols). Monitoring of patients during PS is often substandard, with only two thirds of clinicians using a minimum of blood pressure and pulse oximetry monitors during PS. Commonly used drugs for PS included midazolam, morphine and propofol (91\%, $80 \%$ and $28 \%$, respectively). Propofol (use of which is increasing in the international ED) is more likely to be used by experienced clinicians and those in the private sector. Surprisingly, almost half of clinicians would like propofol
\end{abstract}

P. W. Hodkinson • L. A. Wallis

Division of Emergency Medicine, University of Cape Town,

Cape Town, South Africa

P. W. Hodkinson $(\bowtie)$

P.O. Box 25401, Kampala, Uganda

e-mail: pwhodkinson@gmail.com

M. F. M. James

Department of Anaesthesia, University of Cape Town,

Cape Town, South Africa used on themselves hypothetically, although the majority (62\%) said they had no or limited knowledge of its use and were concerned with its safety.

Conclusions The private sector is generally better serviced for PS than the public sector. Most ED clinicians use morphine and midazolam for PS. However, there is widespread awareness of propofol as an alternative and probably superior PS drug. Recommendations for improving PS include development of general protocols for PS, training of doctors at all levels and optimization of ED facilities and staffing.

Keywords South Africa - Emergency ·

Procedural-sedation $\cdot$ Facilities $\cdot$ Propofol

\section{Introduction}

Currently, no published literature, policy or guidelines on procedural sedation (PS) exist in the emergency department (ED) in South Africa (SA). Emergency medicine is a rapidly developing new speciality in SA with the first specialists graduating in 2007 [1]. The majority of the population (over $80 \%$ ) is serviced by the state-funded, public-sector hospitals, which are often overcrowded and under-resourced [2]. The private sector services those with medical aid (health insurance) and the wealthy.

Procedural sedation is the technique of using drugs to induce a state where a patient will tolerate noxious stimuli, while maintaining his/her own cardio-respiratory function without invasive support and monitoring [3]. Procedures commonly performed in the ED include reduction of dislocations (commonly shoulder, hip, elbow, jaw), reduction of fractures and cardioversion, as well as suturing and certain diagnostic procedures in pediatric patients [4]. There is a continuum of sedation (with ED PS sedation generally 
falling between moderate and deep sedation) that has traditionally been classified as follows [5]:

- Minimal sedation-anxiolysis only; patient responds normally to verbal commands

- Moderate/conscious sedation-patient responds purposefully to commands

- Deep sedation - patient cannot be easily aroused, but responds purposefully to repeated painful stimuli; may require assistance in maintaining airway and spontaneous ventilation

- Very deep sedation/general anesthesia-patient not rousable; impaired cardio-respiratory function requiring support

- Dissociative sedation-cataleptic state induced with ketamine; perception to stimuli is altered, but patients generally maintain airway reflexes and cardio-respiratory function.

Procedural sedation in the ED is now internationally established as a rapid-turnaround, emergency physician-led service, with many excellent review articles available on patient selection (and in particular with regards to fasting criteria for PS), and how, where and who may administer PS [3, 6-9]. Propofol is being advocated internationally as a safe, effective and cost-effective drug for ED PS [10-13]. Standards for safe PS practice require a separate area within the ED, equipped with resuscitation equipment and monitors [13]. Monitoring and documentation of blood pressure, respiratory rate, heart rate and pulse oximetry are mandatory, while the use of capnography and supplemental oxygen administration during PS remain contentious issues as to their clinical significance in the early detection and prevention of hypoxia [14-17].

The aim of this study was to investigate current PS practice in adults in EDs in the Cape Town metropolis. The objective of this investigation is to provide background information necessary for PS training and preparation of protocols for PS in EDs in South Africa, and to consider the potential use of propofol in SA EDs.

\section{Methods}

A cross-sectional descriptive study was undertaken, looking at PS in EDs in the Cape Town metropolis, South Africa. To be included in the study, EDs needed to:

- Be open $24 \mathrm{~h}$ a day

- Be staffed by full-time doctors who primarily practice emergency medicine

- See adult patients.

Practitioners needed to have performed at least one adult PS in the previous 3 months. The ED manager and all full-time medical personnel meeting the inclusion criteria were interviewed by means of two structured questionnaires (one for ED managers, one for clinicians) between April and June 2007.

Data were collected from ED managers, including the size of unit, number and types of PS performed, venue for PS, resuscitation facilities and equipment available, monitoring equipment, personnel (nursing and medical staff numbers and expertise), protocols for PS in place, drugs available for PS, recovery area (monitoring, personnel, duration of recovery), complications of PS, back-up facilities (specialists/ICU/theatre), perceived shortcomings of present PS practice, knowledge and awareness of other drugs and international trends in PS.

Data were collected from clinicians, including work experience, protocols or drug regimes used, personnel involved in PS, monitoring, time to recovery/discharge, side effects and complications experienced, and doctors' satisfaction with present PS practice. Clinicians were also asked which sedation agent they would choose to have used on themselves, and about their knowledge and experience of the use of propofol in ED PS.

Statistical analysis of these data was performed using standard statistical methods: Shapiro-Wilk tests for normal distribution of quantitative data and chi-square testing to determine whether statistically significant association existed between the choice of drug and several independent variables. Analysis of variance (ANOVA) test was performed to determine whether an association existed between estimated recovery time (numerical variable) and the choice of drug. A p-value of less than 0.05 was considered statistically significant. Intercooled STATA 8.0 software (Statacorp LP, College Station, TX) was used.

The study was approved by the Human Research Ethics Committee at the University of Cape Town and conforms to the ethical standards laid down in the 1964 Declaration of Helsinki. To maintain confidentiality, a random identifier was used for entering hospital and clinician-specific data into the database. All participants gave informed consent to their inclusion in the study.

\section{Results}

Thirteen EDs met the inclusion criteria (five public and eight private sector), and 76/89 clinicians were interviewed (85.4\% response rate out of all included clinicians): 28/76 $(36.8 \%)$ were from the private sector and 48/76 (63.2\%) were from the public sector. All ED managers were interviewed face-to-face in their EDs, and the clinicians were interviewed face-to-face or by telephone.

All units performed reduction of shoulder dislocations, and most $(10 / 13,76.9 \%)$ performed elbow and lower-limb 
dislocation reductions. All fracture reductions were referred for orthopedic non-ED management in $6 / 8(75.0 \%)$ private units, while all public units performed upper- and lowerlimb fracture reduction under PS. Cardioversion was performed under PS in 9/13 (69.2\%) EDs.

Table 1 shows the numbers of patients seen per unit and estimated numbers of procedural sedations performed.

The qualifications of medical staff employed in EDs differed substantially between the health sectors: in the private sector there were $4 / 28(10.7 \%)$ consultants, and the remainder were medical officers; while in the public sector there were 2/48 (4.2\%) consultants, 13/48 (27\%) emergency medicine registrars (residents), 6/48 (13\%) community service medical officers (doctors in their first semisupervised year post-internship) and the remainder were medical officers. Experience of medical staff in emergency medicine and anesthetics also varied considerably (Table 2).

Table 3 summarizes the responses by ED managers as to PS facilities and practices, subdivided into private and public sector units.

Non-invasive blood pressure monitoring (NIBP), cardiac (ECG) monitor and pulse oximetry were available in all EDs. The reported level of monitoring used by clinicians during PS is shown in Fig. 1. Use of a minimum of blood pressure and pulse oximetry monitoring was reported by $51 / 76(67.1 \%)$. Of those clinicians who reported use of no monitoring $(5 / 76(6.6 \%))$, all had less than 1 year of ED experience. No clinicians reported regular use of capnography, although this was available in five private units.

Single-clinician performance of both sedation and the procedure was commonly performed according to $66 / 76$ $(86.8 \%)$ of clinicians, and a dedicated nurse was always present during PS according to all private ED clinicians, while in the public sector there was no dedicated nurse according to $40 / 48(83.3 \%)$ of clinicians. Monitoring of patients after PS was usually carried out by a nurse monitoring a single patient in the private sector [20/28 (71.4\%)] and by a nurse monitoring multiple patients in the public sector [47/48 (97.9\%)].

All units had access to morphine, midazolam and ketamine, and 12/13 (92.3\%) had access to etomidate. Fentanyl, the only short-acting opiate widely available, was in $53.8 \%$ of all EDs (7/8 private and 0/5 public EDs). Propofol was available in all but one private unit, and available in two of the public units [9/13 (69.2\%) overall]. Nitrous oxide was accessible in only $4 / 13(30.8 \%)$ of the
EDs. Table 4 summarizes the clinicians' responses to which drugs are commonly used for PS in adults.

All clinicians reported common use of either propofol only 7/76 (9.2\%), midazolam only 55/76 (72.4\%), or both propofol and midazolam 14/76 (18.4\%). These two drugs were used to classify the choice of PS drugs into three groups. Characteristics of clinicians preferring each group of drugs are summarized in Table 5. There was a statistically significant difference in drug use dependent on each variable, demonstrating that more experienced and senior clinicians, as well as those in the private sector, are more likely to use propofol.

Adverse effects were reported in an anecdotal manner and showed no significant difference between the drugs used for PS. Common adverse events were as expectedhypotension, hypoxia and nausea-but there was no useful way to relate the data to incidences. Few serious adverse effects with long-term sequelae were reported.

Clinicians were asked to rate their satisfaction with PS specific to various aspects: time to effect and recovery; ease of sedation and procedure; adverse effects and perceived patient satisfaction. Satisfaction was generally good with all aspects having $80 \%$ or more of clinicians responding that their satisfaction was "fair," "good," or "very good" for each aspect. There was no significant difference between satisfaction and the the drugs used $(\mathrm{p}>0.7)$.

The time taken for patients to recover until they were ready for discharge from the ED was estimated by clinicians to be on average $104 \mathrm{~min}$ (range 18-180 min), with no statistical difference between the three drug groups $(p=0.1435)$.

Use of propofol as an ED PS agent

Clinicians were questioned hypothetically as to their preference for use of a sedation agent on themselves (before any mention of propofol was made to them by the interviewer). Figure 2 summarizes their responses. Of note is that of the $36 / 76(47.4 \%)$ who would prefer propofol PS on themselves, only $17 / 76(47.2 \%)$ of these clinicians report using propofol commonly in their day-to-day practice on patients.

ED managers reported shortcomings of current PS practice in 11/13 (85\%) units. The most frequent issues raised by unit managers were lack of protocols, staffing issues (especially in public-sector hospitals), lack of training

Table 1 Personnel and patient numbers in emergency departments

\begin{tabular}{llll}
\hline & Public $(\mathrm{n}=5)$ & Private $(\mathrm{n}=8)$ & Total $(\mathrm{n}=13)$ \\
\hline Number of patient visits per month, mean (SD; range) & $2,898(1,623 ; 1,000-5,000)$ & $1,694(547 ; 950-2,500)$ & $2,157(1194 ; 950-5,000)$ \\
Estimated number of PS per month, mean (SD; range) & $44.0(20.7 ; 20-70)$ & $41.1(35.4 ; 10-120)$ & $42.2(29.6 ; 10-120)$ \\
\hline
\end{tabular}


Table 2 Experience of ED clinicians in the public and private sectors

\begin{tabular}{llll}
\hline & Working in public-sector ED $(\mathrm{n}=48)$ & Working in private-sector ED $(\mathrm{n}=28)$ & Total $(\mathrm{n}=76)$ \\
\hline $\begin{array}{l}\text { Years post basic medical } \\
\text { qualification, mean (SD; range) }\end{array}$ & $6.6(5.6 ; 1-29)$ & $12.6(8.1 ; 4-30)$ & $8.8(7.20 ; 1-30)$ \\
$\begin{array}{l}\text { Years of experience in ED, mean } \\
(\mathrm{SD} ; \text { range) }\end{array}$ & $2.9(2.7 ; 0.5-12)$ & $7.2(6.3 ; 1-30)$ & $4.5(4.8 ; 0.5-30)$ \\
$\begin{array}{l}\text { Number }(\%) \text { of clinicians with } 6 \\
\text { months or more anesthetics } \\
\text { experience }\end{array}$ & $4 / 48(8.3 \%)$ & $9 / 28(32.1 \%)$ & $13 / 76(17.1 \%)$ \\
\hline
\end{tabular}

in PS, and inadequate area for PS. Clinicians in the public sector complained of insufficient drug availability, staff numbers and equipment, while those in the private sector were largely concerned with drug availability and lack of protocols.

\section{Discussion}

Facilities and personnel

There is a wider range of procedures performed in the public-sector EDs, which may be due to delays in getting patients to theatre for definitive procedures in the public sector, thus resulting in the performance of complex procedures (largely orthopedic) in the ED. Qualifications of clinicians were as anticipated: the public sector employs all of the emergency medicine registrars, as well as the newly qualified community-service medical officers (first year post-internship). Private-sector clinicians would tend to have spent some time and gained experience in the public sector (as well as in anesthetics, which was thought to be an important influence on how ED clinicians might perform PS) before moving to the private EDs, while the public-sector workforce tends to be less experienced with a high turnover of many very junior doctors. Only two units reported having a written PS policy, and in neither case were they up to date or readily available in flow chart or checklist format. Many unit managers claimed to have a verbal PS drug policy, but there were no firm criteria on patient selection (particularly with regards to fasting for PS), monitoring or discharge criteria.

Facilities for PS differed markedly between public- and private-sector units. In the public units, PS is often performed in the general ED area, without immediate access to resuscitation and monitoring equipment, whereas private units tend to have dedicated and well-equipped areas for PS. The lack of resuscitation equipment at the site of PS in public sector units requires urgent consideration because of the risk of life-threatening complications of PS.

All EDs had access to NIBP, pulse oximetry and ECG monitors, although they were not necessarily all functional. Two thirds of clinicians complied with the international minima of BP and pulse oximetry monitoring when performing PS. Of grave concern are the 7\% (largely junior clinicians) who reported routinely performing PS with no

Table 3 Procedural sedation facilities and practice in EDs

\begin{tabular}{|c|c|c|c|}
\hline & \multicolumn{3}{|c|}{ Number (percentage) of unit managers responding "yes" } \\
\hline & Public $(n=5)$ & Private $(\mathrm{n}=8)$ & Total $(n=13)$ \\
\hline Is there a separate area for PS? & $\begin{array}{l}3(60 \%) \text { (2 use } \\
\text { resuscitation area, } 1 \\
\text { specific area) }\end{array}$ & $\begin{array}{l}8(100 \%)(6 \text { use } \\
\quad \text { resuscitation area, } 2 \\
\text { specific area) }\end{array}$ & $11(84.6 \%)$ \\
\hline $\begin{array}{l}\text { Is there adequate resuscitation equipment available } \\
\text { at the bedside? }\end{array}$ & $1(20 \%)$ & $8(100 \%)$ & $9(69.2 \%)$ \\
\hline $\begin{array}{l}\text { Does the unit have a fixed (written) protocol } \\
\text { for PS? }\end{array}$ & 0 & $2(25 \%)$ & $2(15.4 \%)$ \\
\hline $\begin{array}{l}\text { Is } P S \text { performed on pediatric patients } \\
(<13 \text { years old }) \text { ? }\end{array}$ & $3(60 \%)$ & $7(87.5 \%)$ & $10(76.9 \%)$ \\
\hline $\begin{array}{l}\text { Is there } 1: 1 \text { doctor/nurse monitoring until the } \\
\text { patient awakes in all cases? }\end{array}$ & 0 & $7(87.5 \%)$ & $7(53.9 \%)$ \\
\hline $\begin{array}{l}\text { Are you aware of other drug regimens used } \\
\text { internationally for PS which may be superior to your practice? }\end{array}$ & $3(60 \%)$ & $2(25 \%)$ & $5(38.5 \%)$ \\
\hline Do you consider current practice of PS in your unit to be optimal? & $2(40 \%)$ & $3(37.5 \%)$ & $5(38.5 \%)$ \\
\hline
\end{tabular}




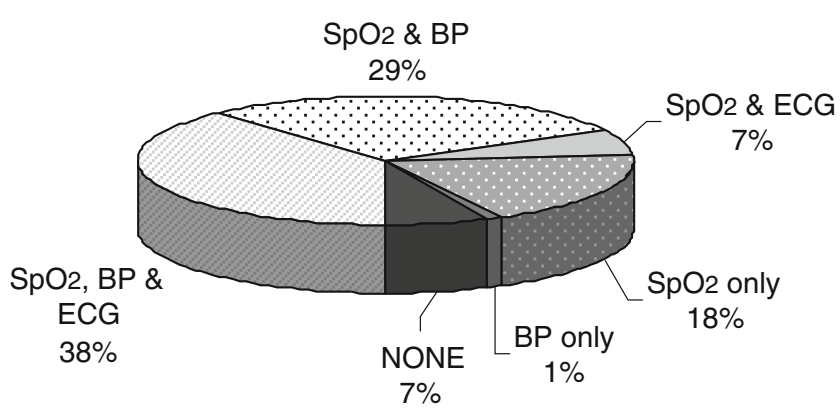

Fig. 1 Clinicans use of various combinations of monitoring during PS $(\mathrm{n}=76) . \mathrm{SpO}_{2}$, pulse oximetry; $\mathrm{BP}$, blood pressure; ECG, cardiac monitor

monitoring, pointing to an urgent need for protocols and training in PS. Capnography, although available in some units, was not used routinely for PS in any unit.

Single-physician PS is becoming the standard of care internationally (with the provision that an appropriately trained assistant is present at all times) [18]. This trend is mirrored in this study, but is likely due to staffing limitations rather than consideration of safety standards. A dedicated nurse is present for less than half of Cape Town's PS. This is an alarming finding from a clinical and medicolegal perspective. Two thirds of patients were monitored by a non-dedicated nurse until they were "awake." Godwin et al. show that most PS side effects and complications occur during PS, and in the first 5 min after PS, but can occur up to 20 min later [3]. Further studies may be necessary to define post-PS monitoring and the personnel and equipment required, as well as the entry and exit criteria for patients being monitored in the South African setting.

Drug choice for PS

Midazolam is widely used in SA EDs for PS, rapid sequence induction and sedation of intubated patients, and

Table 4 Drugs used for PS in EDs in the Cape Town metropolis

\begin{tabular}{lll}
\hline Class of drug & Drug & $\begin{array}{l}\text { Number (percentage) of } \\
\text { clinicians reporting drug } \\
\text { use (n=76) }\end{array}$ \\
\hline Benzodiazepines & Midazolam & $69(90.8 \%)$ \\
& Diazepam & $1(1.3 \%)$ \\
Opiates & Morphine & $61(80.3 \%)$ \\
& Fentanyl & $3(4.0 \%)$ \\
Sedatives (non & Other opiates & $4(5.4 \%)$ \\
benzodiazepine) & Propofol & $21(27.6 \%)$ \\
& Etomidate & $6(7.9 \%)$ \\
Other & Ketamine & $5(6.6 \%)$ \\
\hline
\end{tabular}

has thus become familiar to most ED clinicians. The routine use of antagonists (fluoxetine) to reverse the effects of midazolam (and thus speed up the recovery period) was mentioned by a small number of private-sector clinicians, but there is no evidence to support this as a routine practice. These data confirm that propofol is used, especially by the more senior and experienced senior ED practitioners. The overriding impression from the data is that clinicians are satisfied with present PS practice, and no significance of association was found between the drug groups and the level of satisfaction. In SA, post-procedure monitoring is sub-optimal in terms of staff and facilities, so it could be argued that a drug that provides rapid recovery, such as propofol, is safer than one such as midazolam, which has a prolonged effect possibly resulting in the patient being left poorly monitored in the recovery phase while still at risk of complications (although international studies have found the safety profiles of the two drugs in ED PS to be similar) $[13,19]$. Use of propofol for ED PS will require specific training and skills, and should be part of the core curriculum for emergency medicine specialists in SA as it is elsewhere in the world [10].

It was thought that asking clinicians how they would like PS conducted on themselves would be a good discriminator for which they thought was the ideal PS drug. Almost half of clinicians would request propofol, which contradicts the fact that at most just over a quarter of clinicians report using propofol commonly in their own practice. Reasons for this low usage could include: propofol may not be available to them, they may not be familiar with using the drug, or they may be unhappy with the safety profile of propofol given the circumstances in their own ED (i.e., skills, monitoring and area). Midazolam was a close second choice for $38 \%$ of clinicians to have used on themselves, showing that it is acceptable to many in terms of its safety and effect.

The small number of clinicians with personal experience in using propofol for ED PS shows that it is still largely regarded as an anesthetic drug, and the average ED clinician has little knowledge about its use. There is, however, widespread awareness of PS with propofol, possibly from international exposure and literature. There was some concern that propofol was more expensive to use than other drugs for PS, but this is currently unfounded, with little difference in the costs of propofol and midazolam in SA (personal communication: Victoria Hospital Pharmacy and Constantiaberg Hospital Pharmacy, 2007) and an international study showing cost benefits of the use of propofol [20]. The evidence from this study was not adequate for comparison of time to recovery from agents, but the international literature would suggest time to discharge after propofol PS is less than $1 \mathrm{~h}$, whereas the study mean estimate is $1.7 \mathrm{~h}$ [21]. 
Table 5 Characteristics of clinicians reporting use of propofol only, midazolam and propofol, and midazolam only for PS

\begin{tabular}{|c|c|c|c|c|c|}
\hline Clinicians' characteristics (expressed as \%) & $\begin{array}{l}\text { Propofol } \\
\text { only }(n=7)\end{array}$ & $\begin{array}{l}\text { Propofol and } \\
\text { midazolam }(\mathrm{n}=14)\end{array}$ & $\begin{array}{l}\text { Midazolam } \\
\text { only }(\mathrm{n}=55)\end{array}$ & Total $(n=76)$ & p-value \\
\hline Number in public sector & $0(0 \%)$ & $9(64.3 \%)$ & $39(70.9 \%)$ & $48(63.2 \%)$ & 0.001 \\
\hline Number with 5 years or more experience & $6(85.7 \%)$ & $14(100 \%)$ & $29(52.7 \%)$ & $49(64.5 \%)$ & 0.002 \\
\hline Number with 3 years or more ED experience & $5(71.4 \%)$ & $14(100 \%)$ & $25(45.5 \%)$ & $44(57.9 \%)$ & 0.001 \\
\hline Number with 6 months or more anesthetics experience & $4(57.1 \%)$ & $5(35.7 \%)$ & $4(7.3 \%)$ & $13(17.1 \%)$ & 0.001 \\
\hline Number with international experience & $5(71.4 \%)$ & $8(57.1 \%)$ & $10(18.2 \%)$ & $23(30.3 \%)$ & 0.001 \\
\hline Number of registrars and consultants & $2(28.6 \%)$ & $7(50.0 \%)$ & $8(14.5 \%)$ & $17(22.4 \%)$ & 0.016 \\
\hline
\end{tabular}

The p-value for chi-square test of the association between characteristics of the clinician and drug group used

Unit managers and clinicians were in agreement that better staffing (largely greater numbers and better qualified nurses) would contribute to improved PS practice. Managers thought that protocols and training were priorities, while clinicians were more concerned with drug availability. Lack of equipment was another major issue highlighted by publicsector clinicians.

Further studies in SA are required to investigate pediatric PS as well as specific issues, such as fasting criteria, supplemental oxygen and capnography in PS, as are followup studies to assess the implementation of training and protocol use. It would be useful if these studies were able to better quantify PS practice, thereby giving a measure of PS practice for future research while allowing comparison to international practice.

\section{Limitations}

The data collected in this survey are generally anecdotal estimates; it is therefore impossible to draw firm conclusions from them, and specifically they are not useful for showing the incidences of complications and adverse effects. They are, however, useful for gauging clinicians' opinions and current practices in EDs. The inclusion of EDs with full-time emergency clinicians only has excluded many hospitals in both the public and private sectors. The

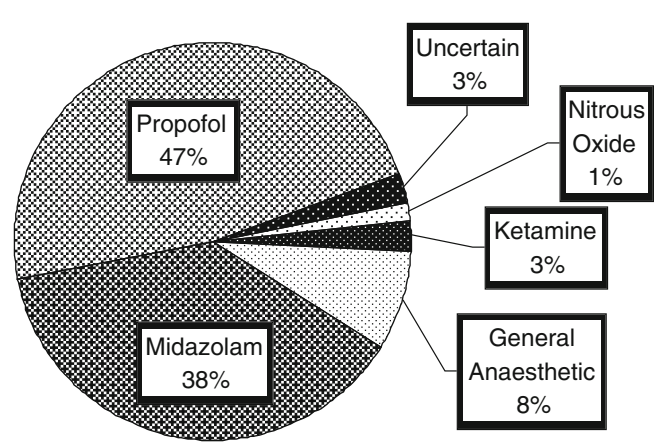

Fig. 2 Clinicians' choice of PS for use on themselves $(n=76)$ excluded units are largely staffed by full-time general practitioners (family practitioners) who do after-hours work in an ED, or part-time-only ED practitioners. Data on the number of PS performed per month were generally estimates by the unit managers as few of the EDs kept specific records in this regard, and therefore may be an under- or over-estimate.

\section{Conclusions}

This study demonstrates many of the shortcomings of PS in Cape Town, although clinicians are generally satisfied with their current practice. These findings are likely common to many developing ED systems around the world. EDs and the new specialists in emergency medicine should be proactive and prepare our EDs for modern PS. Currently, most EDs are not run by specialists, but this will change rapidly over the next few years, with EDs becoming a specialist-run discipline with PS as one of its core capabilities. We need to implement systems to optimize PS such as:

- provision of basic facilities for PS (monitored area with resuscitation equipment)

- guidelines as to personnel requirements (medical and nursing) during and after PS

- formulation of safe and practical protocols that provide guidelines for safe practice and drug use in PS

- training of ED medical personnel (as well as undergraduates) early in their ED training in all aspects of PS.

Acknowledgements The authors would like to thank Dr. Heidi Albert for her support at all stages of this research, including performing all the statistical analysis and reviewing the work. Thanks are also due to the many ED clinicians who willingly participated in the study and gave such insightful data.

This study was presented in partial fulfillment of the MPhil (Emergency Medicine) at the University of Cape Town. 


\section{References}

1. Balfour C (2006) Emergency Medsicine-a new era in South African medicine. S Afr Med J 96:47-48

2. Matsebula T, Willie M (2007) Private Hospitals. In: Harrison S, Bhana R, Ntuli A (eds.) South African Health Review. Health Systems Trust

3. Godwin SA, Caro DA, Wolf SJ et al (2005) Clinical policy: Procedural sedation and analgesia in the emergency department. Ann Emerg Med 45:177-196

4. Sacchetti A, Senula G, Strickland J et al (2007) Procedural Sedation in the Community Emergency Department: Initial results of the ProSCED Registry. Acad Emerg Med 14:41-46

5. Chudnofsky CR, Lozon MM (2005) Procedural sedation and analgesia. In: Rosen's Emergency Medicine. Available via DIALOG. http://rosensemergencymedicine.com/content/book content.cfm?ID=HC188005 Accessed 22/12/2007.

6. Green SM, Roback MG, Miner JR et al (2007) Fasting and emergency department procedural sedation and analgesia: A consensus-based clinical practice advisory. Ann Emerg Med 49:454-461

7. Smally AJ, Nowicki TA (2007) Sedation in the emergency department. Curr Opin Anaesthesiol 20:379-383

8. Miller MA, Levy P, Patel MM (2005) Procedural sedation and analgesia in the emergency department: what are the risks? Emerg Med Clin North Am 23:551-572

9. Bahn EL, Holt KR (2005) Procedural sedation and analgesia: a review and new concepts. Emerg Med Clin North Am 23:503-517

10. Burton JH, Miner JR, Shipley ER et al (2006) Propofol for emergency department procedural sedation and analgesia: a tale of three centers. Acad Emerg Med 13:24-30

11. Green SM, Krauss B (2008) Barriers to propofol use in emergency medicine. Ann Emerg Med 52:392-398

12. Zed PJ, Abu-Laban RB, Chan WW et al (2007) Efficacy, safety and patient satisfaction of propofol for procedural sedation and analgesia in the emergency department: a prospective study. CJEM 9:421-427

13. Miner JR, Burton JH (2007) Clinical Practice Advisory: Emergency Department Procedural Sedation with Propofol. Ann Emerg Med 50:182-187

14. Green SM (2007) Research advances in procedural sedation and analgesia. Ann Emerg Med 49:31-36

15. Krauss B, Hess DR (2007) Capnography for procedural sedation and analgesia in the emergency department. Ann Emerg Med 50:172-181
16. Green SM, Krauss B (2008) Supplemental oxygen during propofol sedation: yes or no (editorial)? Ann Emerg Med 52:9-10

17. Deitch K, Chudnofsky CR, Domenici P (2008) The utility of supplemental oxygen during emergency department procedural sedation with propofol: a randomized controlled trial. Ann Emerg Med 52:1-8

18. Hogan K, Sacchetti A, Aman L et al (2006) The safety of singlephysician procedural sedation in the emergency department. Emerg Med J 23:922-923

19. Hohl CM, Sadatsafavi M, Nosyk B et al (2008) Safety and clinical effectiveness of midazolam versus propofol for procedural sedation in the emergency department: A systematic review. Acad Em Med 15:1-8

20. Hohl CM, Nosyk B, Sadatsafavi M et al (2008) A costeffectiveness analysis of propofol versus midazolam for procedural sedation in the emergency department. Acad Emerg Med 15:32-39

21. Dunn MJG, Mitchell R, De Sousa C et al (2006) Evaluation of propofol and remifentanil for intravenous sedation for reducing shoulder dislocations in the emergency department. Emerg Med J 23:57-58

Peter Hodkinson graduated with a MPhil from the University of Cape Town Emergency Medicine program in 1997. He is currently working at the Makerere University Mulago Hospital in Uganda, trying to help advance Emergency Medicine in Uganda, as well as working as an Honorary Lecturer at the University of Cape Town active in research in the international EM field. This research was conducted in Cape Town in 2007 as part of the requirement for the MPhil (Emergency Medicine) degree.

Prof Mike James is Head of the Department of Anesthetics, University of Cape Town. He has wide research experience and interests and participated in the development of the study with vital input from the anesthetic field.

Prof Lee Wallis is Head of the Division of Emergency Medicine, Stellenbosch University and University of Cape Town, as well as Head of Emergency Medicine, Provincial Government of the Western Cape. He is the first head of the newly formed division and is active in all spheres of the discipline. He has had vital input into this study at all levels. 